\title{
Activity systems modeling as a theoretical lens for social exchange studies
}

\author{
Ernest Jones, III*
}

School of Management, Argosy University, Schaumburg, IL., USA

\section{H R O N I C L E}

\section{Article history:}

Received August 28, 2015

Received in revised format

November 28, 2015

Accepted November 28, 2015

Available online

November 28, 2015

\section{Keywords:}

Social exchange theory

Activity theory

Activity systems modeling

Perceived organizational support

Leader-member exchange

\section{A B S T R A C T}

\begin{abstract}
The social exchange perspective seeks to acknowledge, understand and predict the dynamics of social interactions. Empirical research involving social exchange constructs have grown to be highly technical including confirmatory factor analysis to assess construct distinctiveness and structural equation modeling to assess construct causality. Each study seemingly strives to assess how underlying social exchange theoretic constructs interrelate. Yet despite this methodological depth and resultant explanatory and predictive power, a significant number of studies report findings that, once synthesized, suggest an underlying persistent threat of conceptual or construct validity brought about by a search for epistemological parsimony. Further, it is argued that a methodological approach that embraces inherent complexity such as activity systems modeling facilitates the search for simplified models while not ignoring contextual factors.
\end{abstract}

\section{Introduction}

The social exchange perspective seeks to acknowledge, understand and predict the dynamics of social interactions. Yet, social exchange constructs are varied and complex. Cropanzano and Mitchell's (2005) review of the social exchange literature attests to its complexity. While tracing its roots to the sociology of Blau (1964) and Homans (1958), the field of work has developed into a core of the organizational behavior body of knowledge characterized by a proliferation of studies assessing construct distinctiveness and interrelated cause and effect. Moreover, the researched constructs are not static to the social exchange subfield but has also evolved to include inquiry involving the leadership, group dynamics, participatory behavior subfields of the organizational behavior domain.

This ever expanding boundary is both a testament to the explanatory power of the social exchange

* Corresponding author. Tel: 1-312-804-3103

E-mail address:ernest_jones_iii@stu.argosy.edu(E. Jones III) 
perspective, and a source of concern as the underlying theoretical constructs are extended and applied to ever more workplace contexts. The risk is that the extant empirical, methodological and theoretical literature becomes difficult to navigate. Since study after study pits a subset of social exchange constructs against another and each study has its unique organizational contexts or study subjects, it can become difficult to synthesize a comprehensive model of social exchange.This paper applies activity systems modeling to a subset of the social exchange literature in a way that simultaneously embraces its inherent complexity while striving for epistemological parsimony. The core question addressed herein is whether Engestrom's (1987) activity theory can be utilized as a framework for organizing social exchange theoretic constructs. Specifically, this paper argues that the activity systems approach can be used to develop a model representing the intrapersonal formulation of exchange perspective. Furthermore, it is argued that activity theoretic concepts of conflict and dynamic equilibrium may be useful in not only categorizing existing research but also in proposing new research. Altogether, these premises suggest activity systems modeling is a useful method for embracing the inherent complexity of social exchanges.

First, a review of activity theory is provided. The focus of the narrative, however, is on its philosophical underpinnings in dialectics and utility in modeling the genesis of change rather than the specific instructions on building an activity system. Next, the activity systems theoretic lens is applied to a review of the social exchange literature. The narrative builds a model of what is operationalized as exchange perspective formulation - an intrapersonal viewpoint in which an individual develops a holistic opinion based on the rules of engagement within their organization, their evolved exchange beliefs and their evolved exchange intentions.

\section{The Activity Systems View}

Engestrom (1987) conceptualized activity as the mediated actions of subjects in pursuit of an object. According to the author, actions are mediated by tools, rules, division of labor and community. The culmination of these actions is depicted as a generated activity system (see Fig. 1).

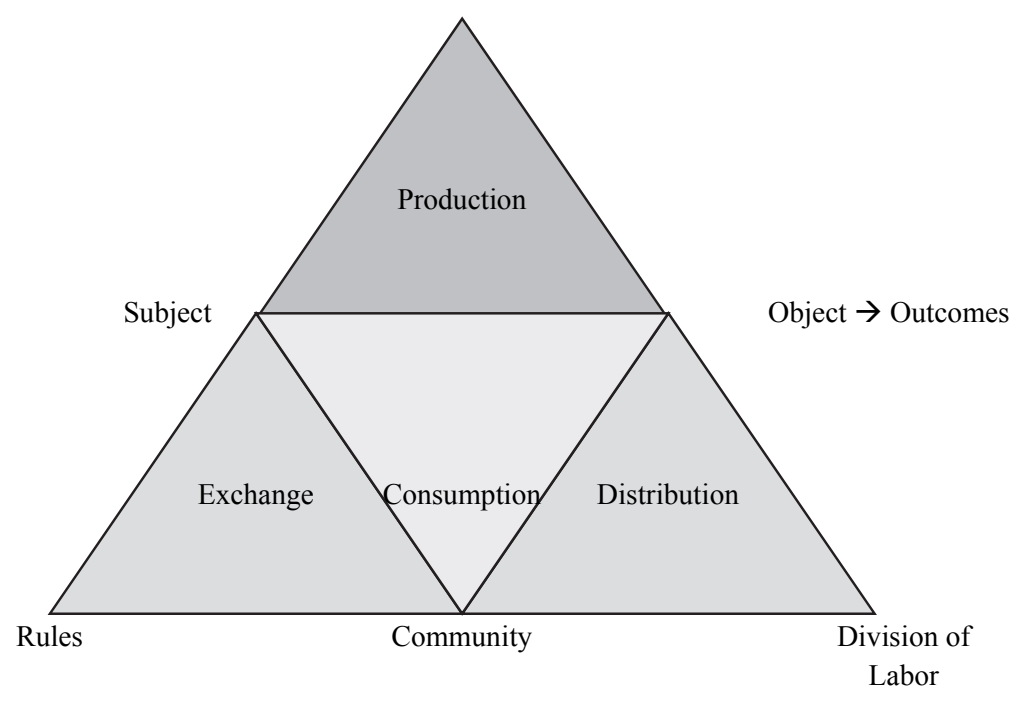

Fig. 1. Generic Activity Systems Diagram.

Additionally, Prenkert (2006) suggests production occurs as tools mediate the subject and object, exchange occurs as rules mediate the subject and community, and distribution occurs as the community and object are mediated by division of labor. Consumption, another activity theoretic construct, occurs as the subject and object are mediated by the community. Finally, although activity is oriented towards an object, there is an anticipated indirect outcome caused by or influenced by the object of the activity. 


\subsection{Dialectical Principles}

Carchedi (2008) reports three fundamental principles of dialectics. The first is that social phenomenal stem both from what is realized and from what may be potentially realized. The second principle suggests that due to this dual nature of reality, what is realized influences what is potential while, simultaneously, what is potential influences what is realized. A direct outcome of this prior proposition is that social phenomena are expected to be under constant change.

Wan's (2012) narrative corroborates Carchedi's (2008) assertions by synthesizing the fundamental premises of dialectics. Namely, Wan's notion of the transformability of quality into quantity and quantity into quality is logically similar to Carchedi's principle of realized and potential attributes of social phenomena. Wan's notion of interpenetration (p. 3) of the realized and the potential is aligned with Carchedi's notion of the influence among the dual dimensions of social phenomena. Wan's findings suggest an additional mechanism of change in that instances of change are simultaneously informed by prior changes.

\subsection{Conflict as a Genesis of Change}

Engestrom (1987) describes four levels of contradiction within and between activity systems. Primary contradictions arise when activity systemic nodes are under inner conflict. Conflicting roles and responsibilities are an example of primary conflict within the division of labor. A secondary contradiction arises, according to Engestrom, when there is a conflict between nodes of an activity system. An example of such conflict occurs when tool use conflicts with the established rules.

In tertiary contradictions, the object of a first activity system is at odds with the object of "a culturally more advanced form of the central activity" (p. 104). Such a case could be when a policy making activity system owned by a functional group delivers new rules resulting in unintended conflict with the overall organization's activity. Finally, in quaternary contradictions, activity systems are in direct conflict with other activity systems.

\section{Social Exchange Perspective Formulation as an Activity System}

Cropanzano and Mitchell (2005) expound on a considerable amount of theoretical inconsistency within the realm of social exchange phenomena. Of particular interest is causality among the various constructs. Another critical part of the authors' review is that during the act of exchange, the quality and character of the transaction can change. Specifically, social transactions coupled with social relationships are espoused as in alignment since the benefits exchanged have similar attributes (e.g. commitment exchanged for support). Likewise, economic transactions coupled with economic relationships are in alignment since the resources of exchange can have tangible value.

Mismatches, however, can exist. Social transactions can be coupled with economic relationships and economic transactions can be coupled with social relationships. These misalignments can be present in workplace context, such as when an individual develop affective commitment, trust, satisfaction as working relationships evolve or, perhaps, when an individual realizes performance rewards are contingent upon the quality and character of her relationship with team members.

If it is accepted that social exchanges moderate participative processes (Cropanzano \& Mitchell, 2005) and that workplace participative processes involve the overt behaviors exhibited by individuals, then the implication is that the health of the workplace environment may be affected by an individual's perception of social exchanges. Thus, the activity theory framework is applied at the intrapersonal level of analysis as a means of investigating the importance of social exchanges. Specifically, the activity systems framework is used to organize various social exchange constructs. Within each apex of the activity system, definitions and justification for constructual positioning will be provided. 


\subsection{The Individual as Subject of the Activity System}

Within this context, the individual is the subject whose objective is to formulate an exchange perspective. Rules includeunderlying societal norms of exchange (Blau, 1964). The community of exchange perspectives is operationalized as organizational culture (Schein, 1996), or, the prevailing exchange perspectives held among community members. The tools of exchange perspective formulation include inward directed social exchange beliefs such as perceived organizational support. The division of labor is operationalized as outward facing exchange intent such as affective commitment, citizenship intent, and withdrawal intent.

\subsection{The Rules of Exchange}

Four constructs in the rules of exchange are applied to the activity systems model. They include reciprocal exchange, negotiated exchange, fairness and justice, and normative commitment.

\subsubsection{Reciprocal Exchange}

Molm (2010) suggests the process of reciprocation has an underlying structure. According to Molm, reciprocity can be modeled as a flow of benefits. In one formulation, reciprocation is conceptualized as the flow of resources among social actors. This flow can be either unilateral or bilateral. Additionally, reciprocity can be either direct or indirect. Direct reciprocity occurs when benefits are exchanged between only two actors. This exchange is unilateral when the flow of benefits is not predefined by time and value unlike bilateral exchange which is reduced to negotiated exchange. Molm's model suggests a third mode of reciprocation in which benefits flow unilaterally from one actor through a network of social actors until the unspecified benefit is returned to the initial actor. The model then proposes causal mechanisms involving the risk of non-reciprocation, the value of the exchange and the potential emergence of competitive behavior and their effect on establishment of trust, affect, and solidarity.

Sparrowe and Liden (1997), like in Molm's (2010) later work, suggest that there exists a reciprocity continuum. The Sparrowe and Liden model of reciprocity, however, relies on alternative structural dimensions. According to the authors, the reciprocity continuum spans from generalized reciprocity at the positive extreme, balanced reciprocity in the middle, and negative reciprocity at the opposing extreme. Within each form of reciprocity, key structural components are the equivalence in benefits returned, the temporal displacement of such benefits and the relative importance of relations or material in the exchange. In generalized reciprocity, the primary concern is for others, the flow of benefits is not expected to be returned with equivalent value or even at all. In balanced reciprocity, the primary concern is mutual flow of benefits coupled with high interest in equal value returned within a reasonable amount of time. Negative reciprocity is characterized by self-interest and the expectation of equal value reciprocated as immediately as possible.

Although separate approaches, the models of reciprocity proposed by Molm (2010) and Sparrowe and Liden (1997) are aligned with what Cropanzano and Mitchell outline as the rules of social exchange. The authors found, through a synthesis of the social exchange literature, reciprocity at the core of social activity. In addition to conceptualizations of reciprocity as interdependent social connections, Cropanzano and Mitchell (2005) found support for reciprocity as cultural norms. Essentially, while reciprocity is theorized as a social relations building process, reciprocity also has normative characteristics. This assertion is relevant to reciprocity's role in the exchange perspective formulation activity system. As a cultural norm and a primary process, reciprocity is firmly established in the rules apex of the Engestrom's (1987) activity systems model as applied in the organizational workplace context. Workplace participants, due to the nature of their day to day involvement within the workplace, are likely to generate reciprocal relationships. However, reciprocity is only one concept that can establish these rules. 


\subsubsection{Negotiated Exchange}

Although negotiated exchanges are embedded within the models of reciprocity reviewed above, the concept deserves additional consideration. In the Sparrowe and Liden (1997) model, negotiated exchange could be characterized as the desire for immediate return of equivalent benefits in the social relationship. Moreover, it can be inferred from the authors' propositions that negotiated exchange could be either balanced (i.e. characterized by mutuality) or negative (i.e. characterized by self-interest). Similarly, negotiated exchange in Molm's (2010) conceptualization is characterized by bilateral and direct exchanges.

Power in negotiated exchanges is an outcome of the structure of the exchange network. According to Barrera (2007), equal power arises when two actors can freely exchange without an intermediary actor. However, when multiple actors are involved concurrent with the possibility that, due to limited resources, exchanges cannot be negotiated among all actors, then the power differences among actors become relevant. A high power actor is one that can never be excluded from the transaction whereas a low power actor may be one that is excluded from an exchange.

Barrera (2007) suggests trust and trustworthiness are critical dimensions of negotiated exchanges and proposes hypotheses that are tested experimentally. One hypothesis suggests an association among repeated exchanges and the emergence of trust and trustworthiness. Trust and trustworthiness, as opined by the author, increases when exchange earnings are high both equal power and unequal power relationships, but are at risk of decreases when low power actors have low earnings resulting from exchanges. A final set of hypotheses suggests trust and trustworthiness increases when a perception of

fairness in exchange by individual actors exists. The primary driver of this view is the perception of reciprocity that may develop due to repeated exchanges.

Negotiated exchanges are relevant to the exchange perspective formulation activity system because of the nature of the workplace processes. In many cases, the goal of the organization is to achieve strategic alignment with its external environment (Daft, 2010). Yet, inputs and outputs of various departments and functional perspectives could be at odds. A rather straightforward example in manufacturing organizations is that the commercial function would prefer robust availability of a company's offerings while the manufacturing function would prefer to minimize costs by making long-running campaigns of standardized models while the financial function would prefer to minimize the amount of cash resources tied up in inventory. Each of these perspectives is in conflict. Thus, negotiated exchanges can be documented as service level agreements among internal functional groups.

\subsubsection{Fairness and Justice}

Cropanzano and Mitchell (2005) allude to the importance of fairness within social transactions and relationships. The concept of fairness can be assessed on three separate dimensions. Procedural justice, according to Rhoades, Eisenberger, and Armeli (2001), is conceptualized as the perceived fairness in the application of organizational policy and the distribution of resources. Distributive justice, a second dimension of fairness, is the perception of fairness in outcomes (Molm et al., 2006). Interactional justice is a close analog to procedural justice but is distinctive (Skarlicki \& Folger, 1997) in that it is the perception of fair treatment relative to the treatment of others.

Rhoades, Eisenberger and Armeli (2001) assert that procedural justice is one of three antecedents to perceived organizational support and associated with the formation of affective organizational commitment. Molm, Collett and Schaefer (2006) proposed a mechanism through which the perception of fairness can be developed. The model suggests as relationships are based more and more on negotiated rather than reciprocal exchange, competition and conflict emerges. This conflict salience directly inhibits the formation of perception of fairness. 


\subsubsection{Normative Commitment}

The commitment construct is yet another multifaceted phenomenon. Meyer and Allen (1991) found three distinctive types of commitment. Affective commitment is the phenomenon in which an individual develops an attachment. Continuance commitment is the phenomenon in which an individual remains attached due to the perception that the costs of severing a reciprocal obligation outweigh the benefits of severing the obligation. Normative commitment, conversely, is the obligation that arises from organizational and societal norms. Meyer et al. (1993) summarize succinctly that affective commitment occurs because individual want to be committed, continuance commitment occurs because the individual feels they must remain commitment, and normative commitment occurs because the individual ought to remain committed.

The notion of an ought (Meyer et al., 1993) implies that normative commitment is an organizational norm and, therefore, is relevant to the discussion of the rules apex of the exchange perspective formulation activity system. Meyer et al. (2002) suggest the antecedents of normative commitment include individual attributes, personal experiences within the organization, and organizational provision directed at enhancing commitment. Consequences of normative commitment, according to the authors, is a reduction in withdrawal intent, improved performance, enhanced citizenship behavioral intent, and enhanced employee wellbeing.

\subsection{Exchange Belief: The Production of Exchange Perspective}

Activity production is facilitated by the use of tools. When extending this framework to the intrapersonal context of the activity of exchange perspective formulation, the production of an exchange perspective is enabled through the use of beliefs. Beliefs, within the exchange perspective formulation activity system, are those phenomena that provide a lens through which an individual interprets events. Relevant constructs are continuance commitment, perceived organizational support, and leader member exchange.

\subsubsection{Continuance Commitment}

Continuance commitment, while found to be a distinctive component of commitment (Meyer \& Allen, 1991; Meyer et al., 1993), has the least statistical power of the three commitment components. Meyer et al. (2002) found that the antecedents of continuance commitment are driven by the alternatives available to organizational members. If an individual feels locked into the organization, then it is surmised that withdrawal intent will be inhibited. The authors, for instance, found age to be a correlate of continuance commitment. However, findings suggest either no impact or a negative impact of continuance commitment on employee wellbeing, performance, citizenship intent.

\subsubsection{Perceived Organizational Support}

Wayne et al. (1997) defined perceived organizational support as the commitment of an individual to an organization derived from the individual's perception of the degree to which the organization values the individual's wellbeing. While similar to organizational commitment, perceived organizational support is asserted by the authors as a wholly distinct concept. While the commitment constructs (e.g. Meyer \& Allen, 1991) is the degree of commitment directed from the individual toward the organization, perceived organizational support is a second order response based on individual interpretation of the organization's directed commitment.

Antecedents of perceived organizational support include, according to Wayne, Shore and Liden (1997), organizationally provided opportunity to enhance career development, promotional rewards and length of service. Outcomes include enhanced affective commitment, increased citizenship intent, and a 
tendency toward slightly increased work performance coupled with decreased withdrawal intent. Rhoades, Eisenberger and Armeli's (2001) investigation into the structural modeling of the antecedents and outcomes of perceived organizational support are fully aligned on affective commitment as an outcome of perceived organizational support. However, the two models differ somewhat in how organizationally provided rewards are operationalized. Moreover, the latter model includes procedural justice and supervisory support as additional antecedents.

Eisenberger et al. (2001) further investigated outcomes of perceived organizational support. Their model suggests perceived organizational support has a direct effect on affective commitment and withdrawal intent. Yet, the authors found mediating variables that influence affective commitment, citizenship intent, performance and withdrawal intent. Specifically, the authors cite Meyer and Allen's (1991) commitment processes in proposing the felt obligation variable which, as proposed by the authors, mediates each of the four outcome variables. A second mediating variable, positive mood, is proposed because, according to the authors, affective commitment and citizenship intent may be enhanced when employees self-report as cheerful or energetic about their workplace.

Tekleab and Chiaburu (2010) further synthesize implications of the perceived organizational support construct. According to the authors, perceived organizational support, psychological contract fulfillment, and trust directed at the organization work together through a latent mediating variable denoted as organizational-focused social exchange. This latent organizationally focused variable results in organizationally directed outcomes including increased job satisfaction, affective commitment, reduced withdrawal intent and enhanced citizenship intent.

\subsubsection{Leader-Member Exchange}

Whereas perceived organizational support is regarded as an individual's response to exchange quality with an organization, leader member exchange focuses on exchange quality among the employeemanager dyad. Graen and Uhl-Bien's (1995) systematic review of the leader-member exchange literature suggests the construct has evolved along multiple dimensions within multiple disciplines. According to the authors, dimensions include leader-based, relationship-based, and follower-based approaches to managing leader member exchanges. Leader-based leadership focuses on overt behavior of the leader and the appropriateness of such behaviors within certain organizational contexts. Relationship based leadership focuses on the flow of reciprocated benefits such as trust and felt obligation between leaders and members. Follower-based leadership takes the members perspective and focuses on the factors that contribute to an individual's motivation and capability for selfmanagement of their own performance. The follower-focused approach to leader member exchange is relevant to the current discussion because of its emphasis on distributed power positions and the potential for the handling of a dilution of a leader's influence due to the influence of the follower group. Graen and Uhl-Bien (1995) plot the evolution of leader member exchange from its original conceptualization as a dyadic linkage among supervisors and employees which invalidated the predominant thinking that leader behaviors should be standardized for each employee relationship. Instead, the vertical dyad linkage studies found support that high quality exchanges were individualized for each leader-member dyad. The implication of these early findings is that due to limited leader time and resources, there would be a distribution of leader-member quality detected across the manager's direct reports as well as throughout the organization. The acceptance of the inherent resource constraint within the leader-focused vertical dyad linkages evolved into research focused on the leader-member relationship. In this stage of development, the focus is on the creation and maintenance of high quality exchange relationships through an emphasis on what Graen and Uhl-Bien calls role making, a process of coaching intended to facilitate the emergence of self-management behaviors and self-management among members. Furthermore, the leader member exchange quality distribution leads to the emergence of in-groups and out-groups - sub groups of members sharing a leader linkage having measurable differences in productivity, satisfaction and withdrawal intent (Graen et al., 1982; Scandura \&Graen, 1984). 
The evolution of follower-based leader-member exchange research is most relevant to the current discussion of leader-member exchange as a tool for the formulation of exchange perspective. Wayne, Shore and Liden's (1997) dyad-based investigation included not only perceived organizational support but also leader-member exchange. Antecedents of member-perceived leader-member exchange quality included manager-assessed social compatibility with the employee, the manager's assessment of the employee's growth potential, and the age of the working relationship. Outcomes included managerassessed performance ratings, manager-assessed citizenship behavior, employee-assessed citizenship intent, and manager- and employee-assessed intent for doing favors.

Tekleab and Chiaburu's (2010) investigation into the form of social exchanges was reviewed earlier for its implications associated with perceived organizational support. The same study also investigated leader-member exchange and found, like with perceived organizational support, that leaders-member exchange works with other variables through a latent mediating variable. In this case, leader-member exchange and trust are antecedents of supervisor focused exchanges. Outcomes of supervisor focused exchanges include in-role performance intentions and citizenship intent. Note that citizenship intent, according to the model, can be directed at either the organization or oriented toward the supervisor.

The preceding discussion alludes not only to the complexity of leader member exchange, its antecedents, outcomes, and interconnectivity with other exchange constructs, but also the complexity of assessing exchange quality along leader, relationship and follower dimensions (Schriesheim et al., 1999). Yet the prevailing measure of exchange quality from the member's perspective was proposed by Scandura and Graen (1984). The relevant aspects of leader-member exchange quality involve the member's perception of a) the leader's satisfaction with the member and the members standing with the leader, b) the degree to which the leader understands the member's workplace issues and needs, c) the degree that the leader recognizes the member's potential, d) the likelihood that the leader will use her positional authority to help solve a member's problem, e) the likelihood that the leader will risk political or social capital to help the member if in tough situation, f) the likelihood that the member will defend the leader's decisions if the leader were not present to do so herself, and g) overall assessment of the member's working relationship with the leader.

\subsection{Exchange Intent: The Distribution of Exchange Perspective}

The discussion thus far has reviewed the rules of exchange perspective formulation. These include the reciprocity norm, normative commitment, and justice. Exchange beliefs, according to the theoretical framework, are those exchange constructs that facilitate the production of exchange perspective by way of assessment of perceived organizational support, leader member exchange quality and continuance commitment. The discussion now transitions to the distribution of exchange perspective. Specifically, affective commitment, citizenship intent, satisfaction, trust, and withdrawal intent are positioned within the exchange perspective formulation activity system.

\subsubsection{Affective Commitment}

Affective commitment has the strongest statistical association among commitment components with social exchange constructs (Cropanzano \& Mitchell, 2005; Jaros, 2007). Rhoades, Eisenberger, and Armeli (2001) found a strong relationship between perceived organizational support and affective commitment. According to structural equation modeling of longitudinal data, the authors conclude that affective commitment is a consequence of perceived organizational support and that perceived organizational support mediates the relationship of procedural justice and affective commitment. These findings provide credence to the activity theoretic positioning of affective commitment within the current study. 


\subsubsection{Organizational Citizenship Intent}

Another outcome of both perceived organizational support and leader-member exchange is the likelihood that an individual will perform extra-role behaviors. Citizenship intention, according to Organ (1988 as cited by Bakhshi et al., 2011), is a multidimensional factor involving an individual's altruistic intentions towards members of the organization and the organization itself, the individual's willingness to embrace suboptimal organizational outcomes, the individual's intention to address and prevent future occurrence of problems within the organization, and the individual's regard for the longterm sustainability of the organization. Deluga (1994) asserts that organizational citizenship intent enacted into behaviors are essential for organizational success.

The discussion has established citizenship as outcomes of both perceived organizational support and leader member exchange. Additional investigations of citizenship intent found that citizenship intent decreases with organizational tenure but is enhanced by normative commitment (Bakhshi et al., 2011), decreases as managerial competence increases although increasing with supervisor trust-building activity and leader member exchange quality (Deluga, 1994, p. 323), and can be both organizationally directed and supervisor directed (Tekleab \& Chiaburu, 2010).

\subsubsection{Satisfaction}

Like with citizenship intent, Tekleab and Chiaburu (2010) assert that the satisfaction construct can be directed both at the organization and the supervisor. Molm's (1991) earlier investigation of satisfaction as part of the social exchange domain found that satisfaction as an outcome of organizational events has a non-linear response to positive and negative outcomes in the presence of power imbalanced social networks.

\subsubsection{Trust}

Many definitions of trust exist. Deluga (1994) operationalizes trust as the expectation that one's commitments are reliable. Molm's (2010, p. 123) definition of trust maintains Deluga's conceptualization of a reliable commitment but insists that the reliable commitment is helpful rather than exploitive.

Trust within the social exchange literature, like most other social exchange constructs, is varied and complex. Cropanzano and Mitchell's (2005) literature review alludes to the importance of trust within social exchanges, an outcome of both transactional and transformation leader behaviors, and suggests trust mediates procedural justice and citizenship intent. Barrera (2007) found empirical support for the notion that repeated negotiated exchange facilitates the emergence of trust when there are no power imbalances. In power imbalanced exchanges, experimental data suggests the high power actor is more likely to display trust.

\subsubsection{Withdrawal Intent}

Another outcome of many models of social exchange, withdrawal intent or the intent to leave the organization is a prominent social exchange construct. Graen et al. (1982) assessed withdrawal intent as an outcome of either the average leadership style and the contemporary conceptualization of leadermember exchange and found leader-member exchange to be the superior predictor. However, Vecchio's (1985) replication of Graen et al.'s earlier study challenged the generalizability of turnover as a predictor of withdrawal intent and suggested that leader member exchange is a predictor of withdrawal intent held among higher level employees rather than those working on the front line. It is notable, however, that both of these studies were completed at the beginning of the information age and that Vecchio's notion of high-level work could be more associated with the degree of knowledge work rather than organizational hierarchy. It is also notable that more recent research involving 
withdrawal intent does not identify leader-member exchange quality as an antecedent of withdrawal intent (Wayne et al., 1997; Eisenberger et al., 2001; Tekleab \& Chiaburu, 2010) and instead focuses on the perceived organizational support as a predictor of withdrawal intent.

\subsection{The Consumption of Exchange Perspectives}

From a purely activity systems theoretic perspective, it can be surmised that the community in which an actor pursues the formulation of a perspective is comprised of the prevailing perspectives held among actors in the activity system. Therefore, it is logical to assert that although these perspectives may be uniquely formulated because of individual experiences and paradigms, it is reasonable to expect that the prevailing perspectives may be shared among actors.

Schein (1996) concluded that there are three prevailing cultures - or patterns of thought-among managers. His narrative on the operators, engineers and executive archetypes provides credence for the above notion that exchange perspectives are held among the community of an activity system. More importantly, Schein himself argues that these archetypes comprise managerial culture.

\section{A Synthesized Framework}

Thus far, this paper has provided justification that social exchange constructs can be arranged into an activity systems model. Fig. 2 provides a visual construct arrangement showing how an individual working within an organizational context formulates an exchange perspective which subsequently leads to outcomes such as participation.

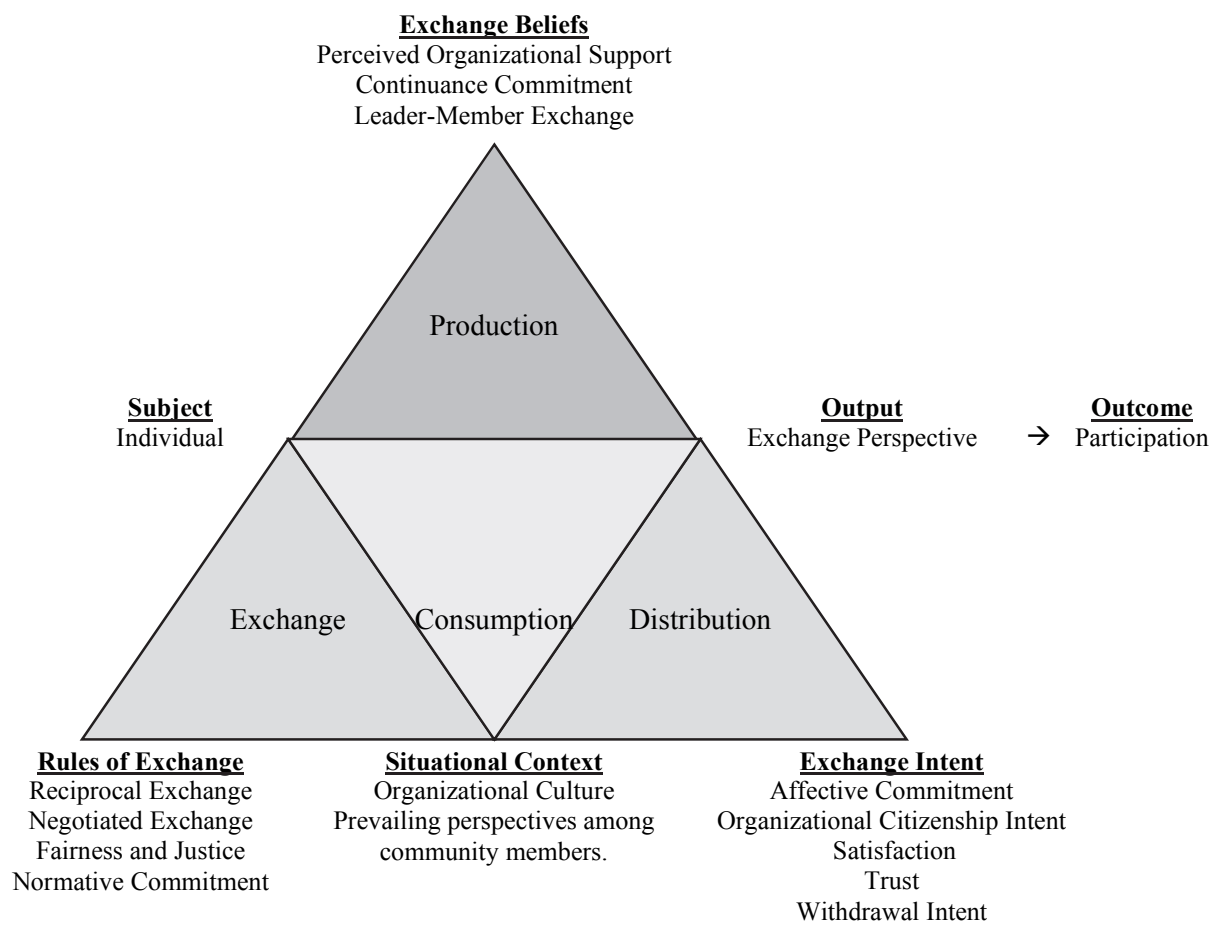

Fig. 2. The Exchange Perspective Formulation Activity System

Within this framework, exchange perspective is generated through the complex interaction of rules (e.g. the individual's assessed meaning and interpretation of reciprocal exchange, normative exchange, fairness, justice and normative commitment), exchange beliefs (e.g. perceived organizational support, continuance commitment, and leader-member exchange), and exchange intentions (e.g. affective commitment, citizenship behavior, satisfaction, trust, and withdrawal intent). 
The utility of the activity theoretic model of exchange perspective formulation stems from its handling of a diverse set of constructs from the extant social exchange literature. The inherent complexity of the organizational context is maintained. Building the framework helps to ensure that important considerations are not inadvertently omitted from the inquiry process. Moreover, adapting the activity systems modeling framework and the sources of conflict can be a useful tool in generating research questions as described in the following examples.

\subsection{Primary and Secondary Conflict}

The above-provided review of the literature covered empirical studies exemplary of primary conflict within this proposed model of exchange perspective formulation. The Wayne, Shore and Liden (1997) hypothesized model of interaction of perceived organizational support and leader member exchange is exemplary of primary conflict. The proposed path model, provided by the authors, shows the constructs in dynamic equilibrium with its specific antecedents and sometimes shared consequences.

The Rhoades, Eisenberger, and Armeli (2001) structural equation model showing the relationship among workplace environments, perceived organizational support, and affective commitment is exemplary of secondary conflict. The exchange perspective formulation activity systems model suggests affective commitment is distributive while perceived organizational support is a beliefbuilding tool. Moreover, the three antecedent constructs to perceived organizational support could be argued as having firm placement within the activity systems model. This paper has already argued for the placement of procedural justice at the rules apex as a subconstruct of fairness and justice. Organizational rewards can be regarded as an artifact of exchange process since organizational rewards could be proceduralized as while simultaneously being under the stakeholdership of the organizational community. However, it is asserted that organizational rewards sit on community apex of the activity systems diagram since organizational rewards have a consumptive quality and are highly likely to be representative of organizational culture. Finally, an individual's perception of supervisor support is firmly placed at the tools apex of the activity systems model. Altogether, the Rhoades, Eisenberger, and Armeli (2001) structural equation model is exemplary of both primary and secondary conflict.

\subsection{Tertiary Conflict}

A notable insight thus far, is that the activity systems model conflict has been internal to the activity systems model itself. In tertiary conflict, the dynamic interactions are outside of the normal bounds of a singular activity system.

Jones (2014) provides an example of tertiary conflict. Fig. 3 shows the genesis of dynamic equilibrium between exchange perspective formulation, a wholly intrapersonal activity system and participation in a workplace activity, a more advanced activity system.

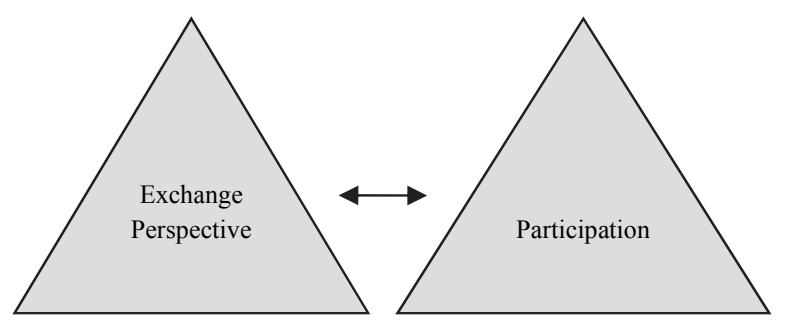

Fig. 3. Two activity systems in Dynamic Equilibrium

Specifically, as noted in Fig. 2, the output of the exchange perspective formulation activity system is a viewpoint on exchanges within the workplace. Jones (2014) argues that although participation is an outcome of exchange perspective formulation, participation is an activity system itself. The conceptual 
framework was the basis of a mixed methods approach in which the exchange perspective formulation activity system influenced an individual-focused participatory behavior activity system. The research study used qualitative methods to find attitudinal specific mechanisms of exchange perspective formulation. These attitudes were used to assess and detect self-reported participatory variables that were synthesized using variables from a culturally more advanced form of the activity. The philosophical underpinning of the study was that the exchange perspective activity system was the dialectical potential while the participatory behavior activity system was the dialectical realized. The study found empirical support for the notion that participatory behavior varies with the model of exchange perspective.

\subsection{Quaternary Conflict}

Activity system models can be generated from more than just the social exchange aspects of the overall organizational behavior field. It is surmisable that an activity system involving constructs from the learning and creativity organizational behavior subfield (Choi, 2012) could be developed as part of an assessment of the literature. A research question could be generated proposing the potential conflict of the exchange perspective formulation activity system and the learning and creativity activity system. A research program could, for example, be designed to assess the interconnectedness of the two activity systems on their shared outcome of intrinsic motivation.

\section{Discussion}

Indeed, it is acknowledged that all research must be tempered by pragmatism. Otherwise, the scope of the inquiry could be unbounded and thus never completed. However, inquiry that ignores contextual factors may yield misleading or misdirected findings. This paper has argued that the use of activity systems modeling may help researchers to not only acknowledge but also to embrace the inherent complexity of social exchange constructs.

The overriding theme of this article is that Engestrom's (1987) conceptualization of an activity systems model is a useful framework for the understanding and study of social exchange constructs. Its major contribution is not only its unique application to organizing constructs but also its ability to facilitate the generation of research questions based on activity theoretic conflict.

While not filling in gaps in the literature, this application of activity theory provides a theoretical lens on a theoretical perspective. In many conceptual and empirical studies, the social exchange perspective is used as a theoretical lens through which research questions can be generated, hypotheses proposed and subsequently tested. Studies on social exchange constructs have evolved into a multitude of discussions surrounding construct distinctiveness and a myriad of models of interrelated causes and effects. Employing the activity systems modeling approach provides additional methodological diversity.

Many social phenomena are only understood with the liberal use of ceteris paribus rules. The field of economics is full of such ceteris paribus laws that claim a certain interrelationship among variables exists if and only if all other variables held constant. Unfortunately, ceteris paribus clauses do not readily exist in reality. Organizational behavior studies and managerial situations are affected by extraneous variables. It is the position of this paper that some extraneous, albeit measurable, variables may not in fact be extraneous, but important moderators or mediators of the social phenomena. The holism of the activity systems modeling approach provides the mechanism for assessing contextual factors that may or may not have an impact on the managerial situation. Indeed, pragmatism is required since a study may not be able to find substantial results using an unlimited variable set. On the contrary, epistemological parsimony should be a goal. However, inconsistent findings, ever-evolving discussions on construct distinctiveness, and a heavy reliance on structural equation modeling - three prominent features of the social exchange literature - could be evidence of a latent conceptual validity concern held by many social exchange theorists due to its not-so-apparent but necessary use of ceteris paribus 
clauses when defining boundaries of a given inquiry.

Use of the activity theoretic lens is not a panacea, however. Perhaps the greatest weakness is errors in application. Spinuzzi (2011), for example, argues that the evolution of the activity systems modeling approach itself has led to difficulty in specifying the object of activity. This ambiguity, according to the author, leads to scope creep and the risk of unbounded research contexts. Within the current investigational context, this difficulty could be most apparent when assessing source of conflict between activity systems. Another commonly known issue within organizational studies is levels of analysis (Klein et al., 1994). However, the activity system view is aptly designed to account for changes in levels...at least qualitatively. Quantitative resolutions of the levels of analysis issue remain problematic. Given these risks and benefits, on balance, the robustness of activity systems modeling approach has been demonstrated in the current application to an intrapersonal process.

Future research into the use of activity theory as a framework for social exchange constructs could be fruitful. The exchange perspective formulation activity system developed herein could be used as a framework for assessing and critiquing existing and future research. The approach could facilitate a transition into alternative methods of theory testing such as Jones' (2014) use of q-methodology (Stephenson, 1953; Brown, 1980) to measure exchange perspective. Furthermore, the dialectical underpinnings of activity theory and its evolution into the conceptualization of primary, secondary, tertiary and quaternary conflict are sources of an unending stream of research applications within, among, and beyond theories of social exchange.

\section{References}

Bakhshi, A., Sharma, A., Kumar, K. (2011). Organizational commitment as a predictor of organizational citizenship behavior. European Journal of Business and Management, 3(4), 78-86.

Barrera, D. (2007). The impact of negotiated exchange on trust and trustworthiness. Social Networks, 29, 508-526. doi:10.1016/j.socnet.2007.03.004.

Blau, P. (1964). Exchange and power in social life. New York:John Wiley.

Brown, S. (1980). Political subjectivity: Applications of Q methodology in political science. New Haven:Yale University Press.

Carchedi, G. (2008). Logic and dialectics in social science, Part I: Dialectics, social phenomena, and non-equilibrium. Critical Sociology, 34(4), 495-523.

Choi, J. (2012). Context and Creativity: The theory of planned behavior as an alternative mechanism. Social Behavior \& Personality: An International Journal, 40(4). 681-692.

Cropanzano, R.,\& Mitchell, M. (2005). Social exchangetheory: An interdisciplinaryreview. Journal of Management, 31(6). 874-900.

Daft, R. (2010). Organization theory and design (10 ${ }^{\text {th }}$ ed.). Mason, OH:South-Western Cengage Learning.

Deluga, R. (1994). Supervisor trust building, leader-member exchange and organizational citizenship behaviour. Journal of Occupational and Organizational Psychology, 67, 315-326.

Eisenberger, R., Armeli, S., Rexwinkel, B., Lynch, P., \& Rhoades, L. (2001). Reciprocation of perceived organizational support. Journal of Applied Psychology, 86(1), 42-51.

Engestrom, Y. (1987). Learning by expanding: An activity theoretical approach to developmental research. Retrieved from http://lchc.ucsd.edu/mca/Paper/Engestrom/expanding/toc.htm

Graen, G., \&Uhl-Bien, M. (1995). Relationship-based approach to leadership : Development of leadermember exchange (LMX) theory of leadership over 25 years : Applying a multi-level multi-domain perspective. Leadership Quarterly, 6(2), 219-247.

Graen, G., Liden, R., \&Hoel, W. (1982). Role of Leadership in the Employee Withdrawal Process. Journal of Applied Psychology, 67(6), 868-872.

Homans, G. (1958). Social Behavior as Exchange. American Journal of Sociology, 63(6), 597-606.

Jaros, S. (2007). Meyer and Allen model of organizational commitment: Measurement issues. Icfai Journal of Organizational Behavior, 6(4), 7-25.

Jones, E. (2014). Five Steps to Navigating the Social Exchange Perspective Landscape. In Lentz, C. 
(Ed.), The Refractive ThinkerVol VIII: Effective Business Practices for Motivation and Communication. Albuquerque, NM:Refractive Thinker Press.

Klein, K., Dansereau, F., Hall, R. (1994). Levels Issues in Theory Development, Data Collection, and Analysis. Academy of Management Review, 19(2), 195-229.

Meyer, J., \& Allen, N. (1991). A three-component conceptualization of organizational commitment. Human Resource Management Review, 1, 61-89.

Meyer, J., Allen, N., \& Smith, C. (1993). Commitment to organizations and occupations: Extension and test of a three-component conceptualization. Journal of Applied Psychology, 78(4), 538-551.

Meyer, J., Stanley, D., Herscovitch, L., \&Topolnytsky, L. (2002). Affective, continuance, and normative commitment to the organization: A meta-analysis of antecedents, correlates, and consequences. Journal of Vocational Behavior, 61, 20-52.

Molm, L. (2010). The structure of reciprocity. Social Psychology Quarterly, 73(2), 119-131.

Molm, L., Collett, J., \&Schaefer, D. (2006). Conflict and fairness in social exchange. Social Forces, 84(4). 2331-2352.

Organ, D.W. (1988). Organizational citizenship behavior: The goodsoldier syndrome. Lexington, MA:Lexington.

Prenkert, F. (2006). A theory of organizing informed by activity theory: The locus of paradox, sources of change, and challenge to management. Journal of Organizational Change, 19(4). 471-490.

Rhoades, L., Eisenberger, R., \&Armeli, S. (2001). Affective commitment to the organization: The contribution of perceived organizational support. Journal of Applied Psychology, 86(5), 825-836.

Roth, W. (2007). Emotion at work: A contribution to third generation cultural historical activity theory. Mind, Culture, and Activity, 14(1-2), 40-63.

Scandura, T., \&Graen, G. (1984). Moderating effects of initial leader-member exchange on the effects of leadership intervention. Journal of Applied Psychology, 69(3), 428-436.

Schein, E. (1996). Culture: The missing concept in organization studies. Administrative Science Quarterly, 41(2), 229-240.

Schriesheim, C., Castro, S., \&Cogliser, C. (1999). Leader-Member Exchange (LMX) Research: A comprehensive review of theory, measurement, and data-analytic practices. Leadership Quarterly, 10(1), 63-113.

Skarlicki, D., \&Folger, R. (1997). Retaliation in the workplace: The roles of distributive, procedural, and interactional justice. Journal of Applied Psychology, 82(3), 434-443.

Sparrowe, R., \&Liden, R. (1997). Process and structure in leader-member exchange. Journal of Management Review, 22(2), 522-552.

Spinuzzi, C. (2011). Losing by Expanding: Corralling the runaway object. Journal of Business and Technical Communication, 25(4), 449-486. DOI: 10.1177/1050651911411040.

Stephenson, W. (1953). The study of behavior: Q-technique and its methodology. Chicago:University of Chicago Press.

Tekleab, A., \&Chiaburu, D. (2010). Social exchange: Empirical examination of form and focus. Journal of Business Research, 64, 460-466.

Vecchio, R. (1985). Predicting employee turnover from leader-member exchange: A failure to replicate. Academy of Management Journal, 28(2), 478-485.

Wan, P. (2012). Dialectics, complexity, and the systemic approach: Toward a critical reconciliation. Philosophy of the Social Sciences, 1-42. DOI: 10.1177/0048393112441974

Wayne, S., Shore, L., \&Liden, R. (1997). Perceived organizational support and leader-member exchange: A social exchange perspective. Academy of Management Journal, 40(1), 82-111.

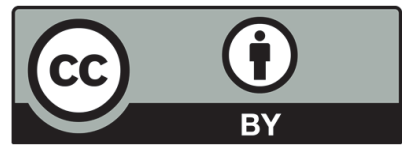

(C) 2016 by the authors; licensee Growing Science, Canada. This is an open access article distributed under the terms and conditions of the Creative Commons Attribution (CC-BY) license (http://creativecommons.org/licenses/by/4.0/). 